\title{
Investigation about wetting ability (surface tension) of water used for preparation of pesticide solutions
}

\author{
Donyo GANCHEV ${ }^{1,2}$
}

Received January 27, 2020; accepted May 17, 2021. Delo je prispelo 27. januarja 2020, sprejeto 17. maja 2021

Investigation about wetting ability (surface tension) of water used for preparation of pesticide solutions

Abstract: The investigation about surface tension of water used for preparation of pesticide solutions reveals it is quite diverse and changeable without any logical correlation towards location, time, and type of water source. Moreover, spraying with solutions with lower surface tension give bigger flow rates due to the lower resistance of fluid to the nozzles. The conducted trials show that plant surfaces with more rough texture require to be sprayed with pesticide solutions with lower surface tension. The wax content of the surfaces has no significant impact on surface tension requirement.

Key words: surface tension; pesticides; plant protection products; sprayers; wetting ability
Preučevanje omočitvene sposobnosti (površinske napetosti) vode za pripravo raztopin pesticidov

Izvleček: Raziskave glede površinske napetosti vode uporabljene za pripravo raztopin pesticidov so odkrile, da je ta zelo raznolika in, da se spreminja brez logične povezave glede na lokacijo, čas in vir vode. Škropljenje $\mathrm{z}$ raztopino $\mathrm{z}$ manjšo površinsko napetostjo daje večje pretoke zaradi manjšega upora tekočine v šobah. Izvedeni poskusi kažejo, da površine rastlin $\mathrm{z}$ bolj grobo teksturo zahtevajo škropljenje $\mathrm{z}$ raztopino pesticidov $\mathrm{z}$ manjšo površinsko napetostjo. Vsebnost voska na površini nima značilnega vpliva glede zahtev o površinski napetosti raztopine pesticidov.

Ključne besede: površinska napetost; pesticidi; pripravki za zaščito rastlin; razpršilci; omočitvena sposobnost

1 Agricultural University - Plovdiv, Bulgaria, Faculty of Plant Protection and Agroecology, Department of Chemistry and Phytopharmacy

2 Corresponding author, e-mail: donyo@abv.bg 


\section{INTRODUCTION}

Using of pesticide solution with low surface tension (good wetting ability) is crucial for achievement of satisfactory level of pesticide effectiveness (Crease \& Thacker, 1991; Ellis et al., 2001,). If the surface tension is too high (wetting ability - too low), pesticide solutions will be at the form of drops on the sprayed surfaces (they will not cover all surface and can be easily dropped down) on one hand and they will not penetrate fully in the rough surfaces from the other. (Ellis et al., 2001). In both cases, the effectiveness of pesticides can be dramatically lowered, especially when pesticide solutions are sprayed in relatively low temperatures (which increase surface tension of the water) and on plant parts with rough surface textures (which require solutions with lower surface tension for full cover in order pesticide solution fully to penetrate into of the surfaces plant tissues - cuticle). The spraying of plants with pesticide solutions over the point of run-off can cause significant risk for contamination of soil and waters (Bergström, 1990).

Traditionally it is expected that common tap water or water from rivers or lakes used for preparing of pesticide solutions have a constant surface tension only slightly depending from temperature (Gittens, 1969; Grisso et al., 1988). Different surface tension means different flow rates and respectively different sprayed dose rates (Matthews, 2008).

The main aim of present investigation was to reveal that the surface tension of the water is highly diverse and changeable and it depends on numerous factors and cannot be predicted in any way unlike common acceptance (Claussen, 1967,; Pallas \& Pethica, 1983; Kalová \& Mareš, 2015;). Also, the common acceptance that plant surfaces with high content of wax like cabbage leaves require to be sprayed with pesticide solution with lower surface tension (better wetting ability) also was wrong - the solutions with lower surface tension is required for surfaces with more rough textures, not with higher wax content (Bartell \& Zuidema, 1936; Hess and Foy, 2000; Wagner et al., 2003). The spraying of solutions with different surface tension can significantly change the flow rates of the sprayers and must be taken into account during treatments with plant protection products (Semiao et.al., 1996; Miller and Ellis, 2000; De Schampheleire et al., 2009)

\section{MATERIALS AND METHODS}

Water samples from different sources in Bulgaria were taken during 2018 year. The sources were:
- tap water from different towns and villages (from different districts of given town / village);

- river and dam lake water from sources situated nearby different towns and villages in Bulgaria;

- well water which although rarely, also is used sometimes for making pesticide solutions (especially during summer drought) from sources situated in different towns and villages;

The 3 samples from one source (location) were taken four times in different time different seasons spring (in May), winter (in February), summer (in August) and autumn ((in October). The surface tension of water samples was established by tensiometer K6 Du Noüy Ring (Macy, 1935), produced by Krüss Scientific, at $24{ }^{\circ} \mathrm{C}$ checked with digital thermometer and average results was presented (used) for each taken sample.

Samples (leaves, barks, fruits) from different plants (mainly agricultural, but also decorative and forest plants) in different $\mathrm{BBCH}$ growth stages were taken and were sprayed with solutions made from distilled water and organosillicone surfactant Silwet L 77, produced by Momentive Performance Materials with different surface tension at $24^{\circ} \mathrm{C}$ (Stevens et al., 1988). The ability of solutions to form even film, to spread easily and uniformly over the treated surfaces (wetting ability) was examined. A liquid with high wetting ability forms a thin, continuous film when it spreads over the surface which allow compete cover of the treated surface with pesticide solutions for maximal effectiveness (maximum cover, exposure and retention) from one side and minimal pollution of the environment (minimal amounts of pesticides fall on soils and waters from treated plants) - from the other (Prado et al., 2016; Zhu et al.,2019). Lower surface tension means better wetting ability (Bonn et al., 2009). Effective wetting ability requires the surface tension of the adhesive $\left(\mathrm{Y}_{\text {adhesive }}\right)$ to

\section{$\gamma_{\mathrm{Lv}} \cos \theta=\gamma_{\mathrm{sv}}-\gamma_{\mathrm{sL}}$}

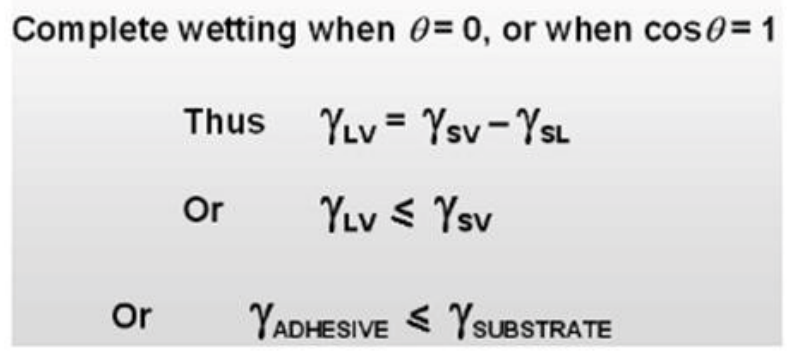

Figure 1: Wetting surface requirement 
be less than or equal to that of the substrate $\left(\mathrm{Y}_{\text {substrate }}\right)$ (Yuan \& Lee, 2013) (Figure. 1)

The flow rate with solution with different surface tension was examined by hand compression Knapsack Sprayer Matabi super green 12 , produced by Goizper. The size of droplets was visually determined by water sensitive papers, produced by Syngenta by method described by Turner and Huntington (1970); Salyani and Fox (1999) and Cerruto et al.(2016) and by digital micrometer (KINEX ABSOLUTE ZERO, 0-25 MM, 0,001MM, DIN 863, IP 65) produced by KINEX Measuring. Nozzles were set to produce spray plum consisting of relatively uniform droplets with diameter of $25 \mu \mathrm{m}$ or $300 \mu \mathrm{m}$.

\section{RESULTS AND DISCUSSION}

The results from conducted trials with measurements of the surface tension from different water sourc- es are presented bellow as tables indicated location of the taken water samples, geographical coordinates, height above the sea level (m), date of taking samples and the average value surface tension of each sample.

\subsection{RESULTS OF TAP WATER ANALYSIS}

The presented Table 1 shows that surface tension of water is absolutely changeable and of upredictable value and can vary in remote places or on a very short distance. For example - town of Plovdiv, Bulgaria, has approximately 102 square kilometers area. The tap water from different residential districts has different surface tension. The same situation is in the towns of Karlovo and Sopot in Central Bulgaria, where the distance between towns is only $4 \mathrm{~km}$ or town of Stamboliiski and village of Joakim Gruevo, where the distance between them is $2 \mathrm{~km}$. The surface tension value of tested tap water was completely different and unpredictable.

Table 1: Surface tension of tap water samples taken in Bulgaria during 2018 in different seasons (winter, spring, summer and autumn)

\begin{tabular}{|c|c|c|c|c|}
\hline Location & Coordinates & $\begin{array}{l}\text { Height above } \\
\text { the sea level } \\
(\mathrm{m})\end{array}$ & $\begin{array}{l}\text { Date of taking } \\
\text { sample }\end{array}$ & $\begin{array}{l}\text { Measured } \\
\text { surface tension } \\
\left(\mathrm{mN} \mathrm{m}^{-1}\right)\end{array}$ \\
\hline Town of Karlovo & $\begin{array}{l}42^{\circ} 63^{\prime} 66^{\prime \prime} \mathrm{N} \\
24^{\circ} 79^{\prime} 98^{\prime \prime} \mathrm{E}\end{array}$ & 452 & 10.02 .2018 & 64 \\
\hline Town of Karlovo & $\begin{array}{l}42^{\circ} 63^{\prime} 66^{\prime \prime} \mathrm{N} \\
24^{\circ} 79^{\prime} 98^{\prime \prime} \mathrm{E}\end{array}$ & 452 & 10.05 .2018 & 58 \\
\hline Town of Karlovo & $\begin{array}{l}42^{\circ} 63^{\prime} 66^{\prime \prime} \mathrm{N} \\
24^{\circ} 79^{\prime} 98^{\prime \prime} \mathrm{E}\end{array}$ & 452 & 10.08 .2018 & 71 \\
\hline Town of Karlovo & $\begin{array}{l}42^{\circ} 63^{\prime} 66^{\prime \prime} \mathrm{N} \\
24^{\circ} 79^{\prime} 98^{\prime \prime} \mathrm{E}\end{array}$ & 452 & 10.10 .2018 & 57 \\
\hline $\begin{array}{l}\text { Village of Vasil Levski, town of } \\
\text { Karlovo district }\end{array}$ & $\begin{array}{l}42^{\circ} 60^{\prime} 80^{\prime \prime} \mathrm{N} \\
24^{\circ} 88^{\prime} 95^{\prime \prime} \mathrm{E}\end{array}$ & 444 & 10.02 .2018 & 78 \\
\hline $\begin{array}{l}\text { Village of Vasil Levski, town of } \\
\text { Karlovo district }\end{array}$ & $\begin{array}{l}42^{\circ} 60^{\prime} 80^{\prime \prime} \mathrm{N} \\
24^{\circ} 88^{\prime} 95^{\prime \prime} \mathrm{E}\end{array}$ & 444 & 10.05 .2018 & 55 \\
\hline $\begin{array}{l}\text { Village of Vasil Levski, town of } \\
\text { Karlovo district }\end{array}$ & $\begin{array}{l}42^{\circ} 60^{\prime} 80^{\prime \prime} \mathrm{N} \\
24^{\circ} 88^{\prime} 95^{\prime \prime} \mathrm{E}\end{array}$ & 444 & 10.08 .2018 & 58 \\
\hline $\begin{array}{l}\text { Village of Vasil Levski, town of } \\
\text { Karlovo district }\end{array}$ & $\begin{array}{l}42^{\circ} 60^{\prime} 80^{\prime \prime} \mathrm{N} \\
24^{\circ} 88^{\prime} 95^{\prime \prime} \mathrm{E}\end{array}$ & 444 & 10.10 .2018 & 64 \\
\hline Town of Stamboliiski & $\begin{array}{l}42^{\circ} 13^{\prime} 25^{\prime \prime} \mathrm{N} \\
24^{\circ} 52^{\prime} 20^{\prime \prime} \mathrm{E}\end{array}$ & 200 & 03.02 .2018 & 63 \\
\hline Town of Stamboliiski & $\begin{array}{l}42^{\circ} 13^{\prime} 25^{\prime \prime} \mathrm{N} \\
24^{\circ} 52^{\prime} 20^{\prime \prime} \mathrm{E}\end{array}$ & 200 & 03.05 .2018 & 72 \\
\hline Town of Stamboliiski & $\begin{array}{l}42^{\circ} 13^{\prime} 25^{\prime \prime} \mathrm{N} \\
24^{\circ} 52^{\prime} 20^{\prime \prime} \mathrm{E}\end{array}$ & 200 & 03.08 .2018 & 71 \\
\hline Town of Stamboliiski & $\begin{array}{l}42^{\circ} 13^{\prime} 25^{\prime \prime} \mathrm{N} \\
24^{\circ} 52^{\prime} 20^{\prime \prime} \mathrm{E}\end{array}$ & 200 & 03.10 .2018 & 65 \\
\hline
\end{tabular}


Village of Joakim Gruevo, town of Stambiliiski district

Village of Joakim Gruevo, town of Stambiliiski district

Village of Joakim Gruevo, town of Stambiliiski district

Village of Joakim Gruevo, town of Stambiliiski district

Town of Jambol

Town of Jambol

Town of Jambol

Town of Jambol

Town of Varna

Town of Varna

Town of Varna

Town of Varna

Agricultural University, town of Plovdiv

Agricultural University, town of Plovdiv

Agricultural University, town of Plovdiv

Agricultural University, town of Plovdiv

town of Plovdiv, residential district

Trakia

town of Plovdiv, residential district

Trakia

town of Plovdiv, residential district

Trakia

town of Plovdiv, residential district

Trakia

town of Plovdiv, residential district

Komatevo

town of Plovdiv, residential district

Komatevo

town of Plovdiv, residential district

Komatevo

town of Plovdiv, residential district

Komatevo

town of Plovdiv, residential district

Karshiaka

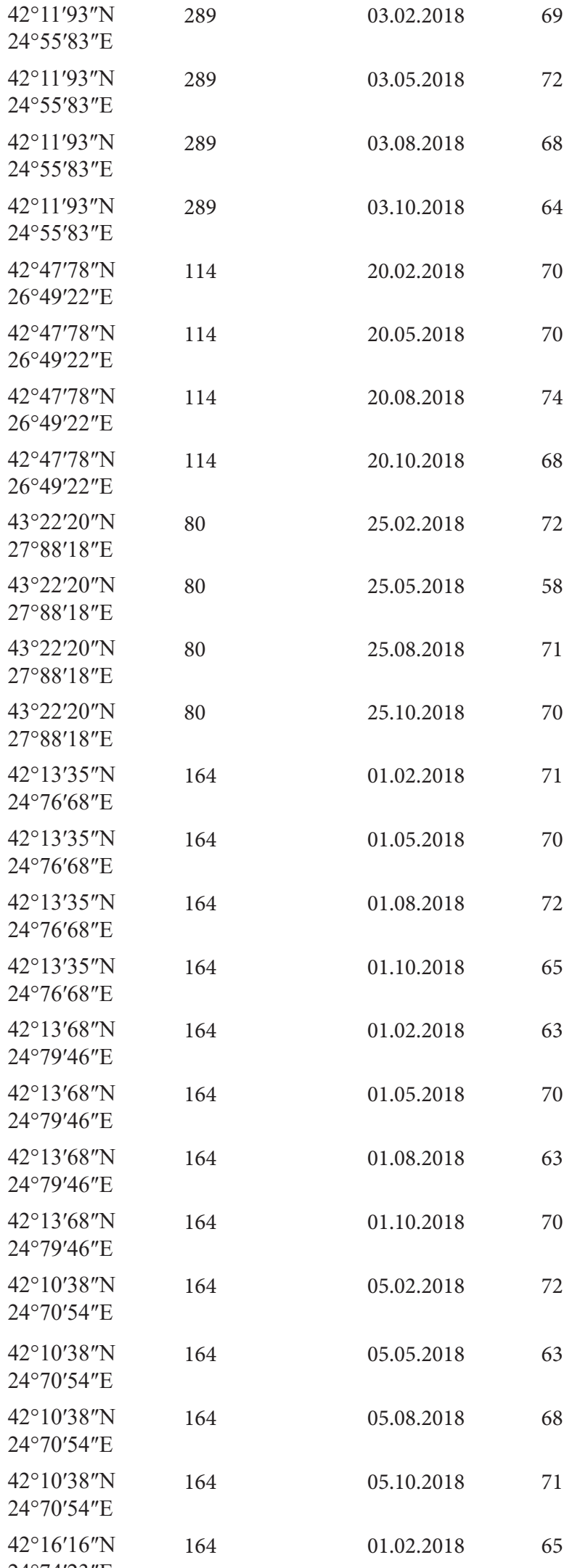

69

72

68

64

70

70

74

68

72

70

71

70

72

65

63

63

70

72

63

68

71

65 


town of Plovdiv, residential district
Karshiaka
town of Plovdiv, residential district
Karshiaka
town of Plovdiv, residential district
Karshiaka
Town of Sopot
Town of Sopot
Town of Sopot
Town of Sopot
Town of Kalofer
Town of Kalofer
Town of Kalofer
Town of Kalofer

town of Plovdiv, residential district

Karshiaka

Karshiaka

town of Plovdiv, residential district

Karshiaka

Town of Sopot

Town of Sopot

Town of Sopot

wn of Kalofer

$\begin{array}{lccc}42^{\circ} 16^{\prime} 16^{\prime \prime} \mathrm{N} & 164 & 01.05 .2018 & 72 \\ 24^{\circ} 74^{\prime} 23^{\prime \prime} \mathrm{E} & & 01.08 .2018 & 70 \\ 42^{\circ} 16^{\prime} 16^{\prime \prime} \mathrm{N} & 164 & 01.10 .2018 & 65 \\ 24^{\circ} 74^{\prime} 23^{\prime \prime} \mathrm{E} & & & \\ 42^{\circ} 16^{\prime} 16^{\prime \prime} \mathrm{N} & 164 & 10.02 .2018 & 58 \\ 24^{\circ} 74^{\prime} 23^{\prime \prime} \mathrm{E} & & & \\ 42^{\circ} 65^{\prime} 04^{\prime \prime} \mathrm{N} & 417 & 10.05 .2018 & 70 \\ 24^{\circ} 76^{\prime} 51^{\prime \prime} \mathrm{E} & & & \\ 42^{\circ} 65^{\prime} 04^{\prime \prime} \mathrm{N} & 417 & & \\ 24^{\circ} 76^{\prime} 51^{\prime \prime} \mathrm{E} & & 10.08 .2018 & 67 \\ 42^{\circ} 65^{\prime} 04^{\prime \prime} \mathrm{N} & 417 & & \\ 24^{\circ} 76^{\prime} 51^{\prime \prime} \mathrm{E} & & & \\ 42^{\circ} 65^{\prime} 04^{\prime \prime} \mathrm{N} & 417 & 10.02 .2018 & 67 \\ 24^{\circ} 76^{\prime} 51^{\prime \prime} \mathrm{E} & & & \\ 42^{\circ} 61^{\prime} 11^{\prime \prime} \mathrm{N} & 666 & 10.05 .2018 & 66 \\ 24^{\circ} 97^{\prime} 78^{\prime \prime} \mathrm{E} & & & \\ 42^{\circ} 61^{\prime} 11^{\prime \prime} \mathrm{N} & 666 & 10.08 .2018 & 69 \\ 24^{\circ} 97^{\prime} 78^{\prime \prime} \mathrm{E} & & & \\ 42^{\circ} 61^{\prime} 11^{\prime \prime} \mathrm{N} & 666 & & \\ 24^{\circ} 97^{\prime} 78^{\prime \prime} \mathrm{E} & & & \\ 42^{\circ} 61^{\prime} 11^{\prime \prime} \mathrm{N} & 666 & & \\ 24^{\circ} 97^{\prime} 78^{\prime \prime} \mathrm{E} & & & \\ \end{array}$

\subsection{RESULTS OF RIVER WATER ANALYSIS}

From Table 2 we can see that just like in the case of tap water, the samples from river water also have different surface tension in different seasons. The water from river Nevolia in the village of Vasil Levski, in February had surface tension of $71 \mathrm{mN} \mathrm{m}^{-1}$, the sample taken from same river, at the same time in the town of Bania, which is situated lie about $7 \mathrm{~km}$ south, had surface tension of $59 \mathrm{mN} \mathrm{m}^{-1}$. The tap water however taken from house $5 \mathrm{~m}$ away from the river Nevolia during February has $78 \mathrm{mN} \mathrm{m}^{-1}$ surface tension. The results confirm the unpredictable nature of the surface tension value towards river waters just like tap waters. Some early investigations reveal direct connection between natural waters contaminations and changes in the surface tension (Pockels, 1893). Certain contaminants can alter water surface tension significantly. The value of surface tension is almost independent of $\mathrm{pH}$ of the water (Sridhar \& Reddy, 1984; Beattie et al., 2014).

\subsection{RESULTS OF LAKE WATER ANALYSIS}

Just like river and tap water samples, the results show absolutely changeable and unpredictable surface tension values for lake. For example, the tap water of the village of Vasil Levski in February had $78 \mathrm{mN} \mathrm{m}^{-1}$ surface tension, the value of this index of river water sample taken from river 5 meters away was $71 \mathrm{mN} \mathrm{m}^{-1}$, the surface tension of dam lake water taken from dam lake Vasil Levski situated approximately $1,8 \mathrm{~km}$ away from the river was $65 \mathrm{mN} \mathrm{m}^{-1}$.

\subsection{RESULTS OF WELL WATER ANALYSIS}

The tap water of the village of Vasil Levski in February had $78 \mathrm{mN} \mathrm{m}^{-1}$ surface tension, the value of this index of river water sample taken from river 5 meters away was $71 \mathrm{mN} \mathrm{m}^{-1}$, the surface tension of dam lake water taken from dam lake Vasil Levski situated approximately $5 \mathrm{~km}$ away from the river was $65 \mathrm{mN} \mathrm{m}^{-1}$, however the water sample taken from well situated 20 $\mathrm{m}$ away from place where river sample and tap water sample were taken, had $57 \mathrm{mN} \mathrm{m}^{-1}$ surface tension. The previously conducted research also confirmed that surface tension of water of soils (underground water) is less than that of pure water (Tschapek et al., 1978). From the results for well waters in present study, is obvious that this value sometimes is less, sometimes is bigger. For sure is completely unpredictable and changeable. However, in present study only freshwater sources were examined. Other study shows that according to the seawater sample analysis there is similar uncertainty in prediction on surface tension as sweet water (Nayar et al., 2014). 
Table 2: Surface tension of river water samples taken in Bulgaria during 2018 in different seasons (winter, spring, summer and autumn)

\begin{tabular}{|c|c|c|c|c|}
\hline Location & Coordinates & $\begin{array}{l}\text { Height above the } \\
\text { sea level }(\mathrm{m})\end{array}$ & $\begin{array}{l}\text { Date of taking } \\
\text { sample }\end{array}$ & $\begin{array}{l}\text { Measured } \\
\text { surface tension } \\
\left(\mathrm{mN} \mathrm{m}^{-1}\right)\end{array}$ \\
\hline $\begin{array}{l}\text { River Nevolia, village of Vasil } \\
\text { Levski, town of Karlovo district }\end{array}$ & $\begin{array}{l}42^{\circ} 60^{\prime} 80^{\prime \prime} \mathrm{N} \\
24^{\circ} 88^{\prime} 99^{\prime \prime} \mathrm{E}\end{array}$ & 444 & 10.02 .2018 & 71 \\
\hline $\begin{array}{l}\text { River Nevolia, village of Vasil } \\
\text { Levski, town of Karlovo district }\end{array}$ & $\begin{array}{l}42^{\circ} 60^{\prime} 80^{\prime \prime} \mathrm{N} \\
24^{\circ} 88^{\prime} 99^{\prime \prime} \mathrm{E}\end{array}$ & 444 & 10.05.2018 & 56 \\
\hline $\begin{array}{l}\text { River Nevolia, village of Vasil } \\
\text { Levski, town of Karlovo district }\end{array}$ & $\begin{array}{l}42^{\circ} 60^{\prime} 80^{\prime \prime} \mathrm{N} \\
24^{\circ} 88^{\prime} 99^{\prime \prime} \mathrm{E}\end{array}$ & 444 & 10.08 .2018 & 74 \\
\hline $\begin{array}{l}\text { River Nevolia, village of Vasil } \\
\text { Levski, town of Karlovo district }\end{array}$ & $\begin{array}{l}42^{\circ} 60^{\prime} 80^{\prime \prime} \mathrm{N} \\
24^{\circ} 88^{\prime} 99^{\prime \prime} \mathrm{E}\end{array}$ & 444 & 10.10 .2018 & 62 \\
\hline River Nevolia, town of Banija & $\begin{array}{l}42^{\circ} 56^{\prime} 33^{\prime \prime} \mathrm{N} \\
24^{\circ} 83^{\prime} 02^{\prime \prime} \mathrm{E}\end{array}$ & 295 & 10.02 .2018 & 59 \\
\hline River Nevolia, town of Banija & $\begin{array}{l}42^{\circ} 56^{\prime} 33^{\prime \prime} \mathrm{N} \\
24^{\circ} 83^{\prime} 02^{\prime \prime} \mathrm{E}\end{array}$ & 295 & 10.05 .2018 & 63 \\
\hline River Nevolia, town of Banija & $\begin{array}{l}42^{\circ} 56^{\prime} 33^{\prime \prime} \mathrm{N} \\
24^{\circ} 83^{\prime} 02^{\prime \prime} \mathrm{E}\end{array}$ & 295 & 10.08 .2018 & 68 \\
\hline River Nevolia, town of Banija & $\begin{array}{l}42^{\circ} 56^{\prime} 33^{\prime \prime} \mathrm{N} \\
24^{\circ} 83^{\prime} 02^{\prime \prime} \mathrm{E}\end{array}$ & 295 & 10.10 .2018 & 72 \\
\hline River Strjama, town of Banija & $\begin{array}{l}42^{\circ} 54^{\prime} 20^{\prime \prime} \mathrm{N} \\
24^{\circ} 82^{\prime} 08^{\prime \prime} \mathrm{E}\end{array}$ & 295 & 10.02 .2018 & 60 \\
\hline River Nevolia, town of Banija & $\begin{array}{l}42^{\circ} 56^{\prime} 33^{\prime \prime} \mathrm{N} \\
24^{\circ} 83^{\prime} 02^{\prime \prime} \mathrm{E}\end{array}$ & 295 & 10.05 .2018 & 74 \\
\hline River Nevolia, town of Banija & $\begin{array}{l}42^{\circ} 56^{\prime} 33^{\prime \prime} \mathrm{N} \\
24^{\circ} 83^{\prime} 02^{\prime \prime} \mathrm{E}\end{array}$ & 295 & 10.08 .2018 & 75 \\
\hline River Nevolia, town of Banija & $\begin{array}{l}42^{\circ} 56^{\prime} 33^{\prime \prime} \mathrm{N} \\
24^{\circ} 83^{\prime} 02^{\prime \prime} \mathrm{E}\end{array}$ & 295 & 10.10 .2018 & 57 \\
\hline River Jantra, town of Gabrovo & $\begin{array}{l}42^{\circ} 89^{\prime} 09^{\prime \prime} \mathrm{N} \\
25^{\circ} 32^{\prime} 42^{\prime \prime} \mathrm{E}\end{array}$ & 392 & 27.02 .2018 & 71 \\
\hline River Jantra, town of Gabrovo & $\begin{array}{l}42^{\circ} 89^{\prime} 09^{\prime \prime} \mathrm{N} \\
25^{\circ} 32^{\prime} 42^{\prime \prime} \mathrm{E}\end{array}$ & 392 & 27.05 .2018 & 68 \\
\hline River Jantra, town of Gabrovo & $\begin{array}{l}42^{\circ} 89^{\prime} 09^{\prime \prime} \mathrm{N} \\
25^{\circ} 32^{\prime} 42^{\prime \prime} \mathrm{E}\end{array}$ & 392 & 27.08.2018 & 65 \\
\hline River Jantra, town of Gabrovo & $\begin{array}{l}42^{\circ} 89^{\prime} 09^{\prime \prime} \mathrm{N} \\
25^{\circ} 32^{\prime} 42^{\prime \prime} \mathrm{E}\end{array}$ & 392 & 27.10 .2018 & 65 \\
\hline River Osam, town of Trojan & $\begin{array}{l}42^{\circ} 91^{\prime} 52^{\prime \prime} \mathrm{N} \\
24^{\circ} 71^{\prime} 01^{\prime \prime} \mathrm{E}\end{array}$ & 380 & 27.02 .2018 & 64 \\
\hline River Osam, town of Trojan & $\begin{array}{l}42^{\circ} 91^{\prime} 52^{\prime \prime} \mathrm{N} \\
24^{\circ} 71^{\prime} 01^{\prime \prime} \mathrm{E}\end{array}$ & 380 & 27.05 .2018 & 65 \\
\hline River Osam, town of Trojan & $\begin{array}{l}42^{\circ} 91^{\prime} 52^{\prime \prime} \mathrm{N} \\
24^{\circ} 71^{\prime} 01^{\prime \prime} \mathrm{E}\end{array}$ & 380 & 27.08 .2018 & 55 \\
\hline River Osam, town of Trojan & $\begin{array}{l}42^{\circ} 91^{\prime} 52^{\prime \prime} \mathrm{N} \\
24^{\circ} 71^{\prime} 01^{\prime \prime} \mathrm{E}\end{array}$ & 380 & 27.10 .2018 & 65 \\
\hline
\end{tabular}


Table 3: Surface tension of dam lake water samples taken in Bulgaria during 2018 in different seasons (winter, spring, summer and autumn)

\begin{tabular}{|c|c|c|c|c|}
\hline Location & Coordinates & $\begin{array}{l}\text { Height above the } \\
\text { sea level } \\
(\mathrm{m})\end{array}$ & Date of taking sampels & $\begin{array}{l}\text { Measured } \\
\text { surface tension } \\
\left(\mathrm{mN} \mathrm{m}^{-1}\right)\end{array}$ \\
\hline $\begin{array}{l}\text { Dam lake Vasil Levski, village of } \\
\text { Vasil Levski, Karlovo district }\end{array}$ & $\begin{array}{l}42^{\circ} 61^{\prime} 48^{\prime \prime} \mathrm{N} \\
24^{\circ} 91^{\prime} 16^{\prime \prime} \mathrm{E}\end{array}$ & 444 & 10.02 .2018 & 65 \\
\hline $\begin{array}{l}\text { Dam lake Vasil Levski, village of } \\
\text { Vasil Levski, Karlovo district }\end{array}$ & $\begin{array}{l}42^{\circ} 61^{\prime} 48^{\prime \prime} \mathrm{N} \\
24^{\circ} 91^{\prime} 16^{\prime \prime} \mathrm{E}\end{array}$ & 444 & 10.05 .2018 & 60 \\
\hline $\begin{array}{l}\text { Dam lake Vasil Levski, village of } \\
\text { Vasil Levski, Karlovo district }\end{array}$ & $\begin{array}{l}42^{\circ} 61^{\prime} 48^{\prime \prime} \mathrm{N} \\
24^{\circ} 91^{\prime} 16^{\prime \prime} \mathrm{E}\end{array}$ & 444 & 10.08 .2018 & 67 \\
\hline $\begin{array}{l}\text { Dam lake Vasil Levski, village of } \\
\text { Vasil Levski, Karlovo district }\end{array}$ & $\begin{array}{l}42^{\circ} 61^{\prime} 48^{\prime \prime} \mathrm{N} \\
24^{\circ} 91^{\prime} 16^{\prime \prime} \mathrm{E}\end{array}$ & 444 & 10.10 .2018 & 70 \\
\hline Dam lake Murla, town of Sopot & $\begin{array}{l}42^{\circ} 64^{\prime} 74^{\prime \prime} \mathrm{N} \\
24^{\circ} 77^{\prime} 21^{\prime \prime} \mathrm{E}\end{array}$ & 417 & 10.02 .2018 & 55 \\
\hline Dam lake Murla, town of Sopot & $\begin{array}{l}42^{\circ} 64^{\prime} 74^{\prime \prime} \mathrm{N} \\
24^{\circ} 77^{\prime} 21^{\prime \prime} \mathrm{E}\end{array}$ & 417 & 10.05 .2018 & 55 \\
\hline Dam lake Murla, town of Sopot & $\begin{array}{l}42^{\circ} 64^{\prime} 74^{\prime \prime} \mathrm{N} \\
24^{\circ} 77^{\prime} 21^{\prime \prime} \mathrm{E}\end{array}$ & 417 & 10.08 .2018 & 65 \\
\hline Dam lake Murla, town of Sopot & $\begin{array}{l}42^{\circ} 64^{\prime} 74^{\prime \prime} \mathrm{N} \\
24^{\circ} 77^{\prime} 21^{\prime \prime} \mathrm{E}\end{array}$ & 417 & 10.10 .2018 & 68 \\
\hline $\begin{array}{l}\text { Dam lake Kovatchevo, village of Kovatchevo, town of } \\
\text { Stara Zagora district }\end{array}$ & $\begin{array}{l}42^{\circ} 21^{\prime} 83^{\prime \prime} \mathrm{N} \\
24^{\circ} 20^{\prime} 90^{\prime \prime} \mathrm{E}\end{array}$ & 133 & 20.02 .2018 & 59 \\
\hline $\begin{array}{l}\text { Dam lake Kovatchevo, village of Kovatchevo, town of } \\
\text { Stara Zagora district }\end{array}$ & $\begin{array}{l}42^{\circ} 21^{\prime} 83^{\prime \prime} \mathrm{N} \\
24^{\circ} 20^{\prime} 90^{\prime \prime} \mathrm{E}\end{array}$ & 133 & 20.05 .2018 & 70 \\
\hline $\begin{array}{l}\text { Dam lake Kovatchevo, village of Kovatchevo, town of } \\
\text { Stara Zagora district }\end{array}$ & $\begin{array}{l}42^{\circ} 21^{\prime} 83^{\prime \prime} \mathrm{N} \\
24^{\circ} 20^{\prime} 90^{\prime \prime} \mathrm{E}\end{array}$ & 133 & 20.08 .2018 & 67 \\
\hline $\begin{array}{l}\text { Dam lake Kovatchevo, village of Kovatchevo, town of } \\
\text { Stara Zagora district }\end{array}$ & $\begin{array}{l}42^{\circ} 21^{\prime} 83^{\prime \prime} \mathrm{N} \\
24^{\circ} 20^{\prime} 90^{\prime \prime} \mathrm{E}\end{array}$ & 133 & 20.10 .2018 & 65 \\
\hline Dam lake Zrebchevo, town of Nikolaevo & $\begin{array}{l}42^{\circ} 63^{\prime} 19^{\prime \prime} \mathrm{N} \\
25^{\circ} 84^{\prime} 95^{\prime \prime} \mathrm{E}\end{array}$ & 274 & 20.02 .2018 & 58 \\
\hline Dam lake Zrebchevo, town of Nikolaevo & $\begin{array}{l}42^{\circ} 63^{\prime} 19^{\prime \prime} \mathrm{N} \\
25^{\circ} 84^{\prime} 95^{\prime \prime} \mathrm{E}\end{array}$ & 274 & 20.05 .2018 & 68 \\
\hline Dam lake Zrebchevo, town of Nikolaevo & $\begin{array}{l}42^{\circ} 63^{\prime} 19^{\prime \prime} \mathrm{N} \\
25^{\circ} 84^{\prime} 95^{\prime \prime} \mathrm{E}\end{array}$ & 274 & 20.08 .2018 & 57 \\
\hline Dam lake Zrebchevo, town of Nikolaevo & $\begin{array}{l}42^{\circ} 63^{\prime} 19^{\prime \prime} \mathrm{N} \\
25^{\circ} 84^{\prime} 95^{\prime \prime} \mathrm{E}\end{array}$ & 274 & 20.10 .2018 & 73 \\
\hline $\begin{array}{l}\text { Dam Lake Radetski, village of } \\
\text { Radetski, town of Nova Zagora District }\end{array}$ & $\begin{array}{l}42^{\circ} 28^{\prime} 51^{\prime \prime} \mathrm{N} \\
26^{\circ} 09^{\prime} 65^{\prime \prime} \mathrm{E}\end{array}$ & 156 & 20.02 .2018 & 66 \\
\hline $\begin{array}{l}\text { Dam Lake Radetski, village of } \\
\text { Radetski, town of Nova Zagora District }\end{array}$ & $\begin{array}{l}42^{\circ} 28^{\prime} 51^{\prime \prime} \mathrm{N} \\
26^{\circ} 09^{\prime} 65^{\prime \prime} \mathrm{E}\end{array}$ & 156 & 20.05 .2018 & 70 \\
\hline $\begin{array}{l}\text { Dam Lake Radetski, village of } \\
\text { Radetski, town of Nova Zagora District }\end{array}$ & $\begin{array}{l}42^{\circ} 28^{\prime} 51^{\prime \prime} \mathrm{N} \\
26^{\circ} 09^{\prime} 65^{\prime \prime} \mathrm{E}\end{array}$ & 156 & 20.08 .2018 & 70 \\
\hline $\begin{array}{l}\text { Dam Lake Radetski, village of } \\
\text { Radetski, town of Nova Zagora District }\end{array}$ & $\begin{array}{l}42^{\circ} 28^{\prime} 51^{\prime \prime} \mathrm{N} \\
26^{\circ} 09^{\prime} 65^{\prime \prime} \mathrm{E}\end{array}$ & 156 & 20.10 .2018 & 65 \\
\hline $\begin{array}{l}\text { Dam Lake Matza, village Matza, town of } \\
\text { Polski Gradetz district }\end{array}$ & $\begin{array}{l}42^{\circ} 22^{\prime} 14^{\prime \prime} \mathrm{N} \\
26^{\circ} 15^{\prime} 20^{\prime \prime} \mathrm{E}\end{array}$ & 190 & 20.02 .2018 & 71 \\
\hline $\begin{array}{l}\text { Dam Lake Matza, village Matza, town of } \\
\text { Polski Gradetz district }\end{array}$ & $\begin{array}{l}42^{\circ} 22^{\prime} 14^{\prime \prime} \mathrm{N} \\
26^{\circ} 15^{\prime} 20^{\prime \prime} \mathrm{E}\end{array}$ & 190 & 20.05 .2018 & 74 \\
\hline $\begin{array}{l}\text { Dam Lake Matza, village Matza, town of } \\
\text { Polski Gradetz district }\end{array}$ & $\begin{array}{l}42^{\circ} 22^{\prime} 14^{\prime \prime} \mathrm{N} \\
26^{\circ} 15^{\prime} 20^{\prime \prime} \mathrm{E}\end{array}$ & 190 & 20.08 .2018 & 71 \\
\hline $\begin{array}{l}\text { Dam Lake Matza, village Matza, town of } \\
\text { Polski Gradetz district }\end{array}$ & $\begin{array}{l}42^{\circ} 22^{\prime} 14^{\prime \prime} \mathrm{N} \\
26^{\circ} 15^{\prime} 20^{\prime \prime} \mathrm{E}\end{array}$ & 190 & 20.10 .2018 & 70 \\
\hline
\end{tabular}


Table 4: Surface tension of well water samples taken in Bulgaria during 2018 in different seasons (winter, spring, summer and autumn)

\begin{tabular}{|c|c|c|c|c|}
\hline Location & Coordinates & $\begin{array}{l}\text { Height above the sea } \\
\text { level }(\mathrm{m})\end{array}$ & $\begin{array}{l}\text { Date of taking } \\
\text { sampels }\end{array}$ & $\begin{array}{l}\text { Measured } \\
\text { surface tension } \\
\left(\mathrm{mN} \mathrm{m}^{-1}\right)\end{array}$ \\
\hline $\begin{array}{l}\text { Village of Radetski, town of Nova } \\
\text { Zagora district }\end{array}$ & $\begin{array}{l}42^{\circ} 28^{\prime} 39^{\prime \prime} \mathrm{N} \\
26^{\circ} 11^{\prime} 45^{\prime \prime} \mathrm{E}\end{array}$ & 156 & 20.02.2018 & 71 \\
\hline $\begin{array}{l}\text { Village of Radetski, town of } \\
\text { Nova Zagora district }\end{array}$ & $\begin{array}{l}42^{\circ} 28^{\prime} 39^{\prime \prime} \mathrm{N} \\
26^{\circ} 11^{\prime} 45^{\prime \prime} \mathrm{E}\end{array}$ & 156 & 20.05 .2018 & 58 \\
\hline $\begin{array}{l}\text { Village of Radetski, town of } \\
\text { Nova Zagora district }\end{array}$ & $\begin{array}{l}42^{\circ} 28^{\prime} 39^{\prime \prime} \mathrm{N} \\
26^{\circ} 11^{\prime} 45^{\prime \prime} \mathrm{E}\end{array}$ & 156 & 20.08 .2018 & 72 \\
\hline $\begin{array}{l}\text { Village of Radetski, town of } \\
\text { Nova Zagora district }\end{array}$ & $\begin{array}{l}42^{\circ} 28^{\prime} 39^{\prime \prime} \mathrm{N} \\
26^{\circ} 11^{\prime} 45^{\prime \prime} \mathrm{E}\end{array}$ & 156 & 20.10 .2018 & 70 \\
\hline $\begin{array}{l}\text { Village of Vasil Levski, town of } \\
\text { Karlovo district }\end{array}$ & $\begin{array}{l}42^{\circ} 60^{\prime} 84^{\prime \prime} \mathrm{N} \\
24^{\circ} 88^{\prime} 83^{\prime \prime} \mathrm{E}\end{array}$ & 444 & 10.02 .2018 & 57 \\
\hline $\begin{array}{l}\text { Village of Vasil Levski, town of } \\
\text { Karlovo district }\end{array}$ & $\begin{array}{l}42^{\circ} 60^{\prime} 84^{\prime \prime} \mathrm{N} \\
24^{\circ} 88^{\prime} 83^{\prime \prime} \mathrm{E}\end{array}$ & 444 & 10.05 .2018 & 68 \\
\hline $\begin{array}{l}\text { Village of Vasil Levski, town of } \\
\text { Karlovo district }\end{array}$ & $\begin{array}{l}42^{\circ} 60^{\prime} 84^{\prime \prime} \mathrm{N} \\
24^{\circ} 88^{\prime} 83^{\prime \prime} \mathrm{E}\end{array}$ & 444 & 10.08 .2018 & 55 \\
\hline $\begin{array}{l}\text { Village of Vasil Levski, town of } \\
\text { Karlovo district }\end{array}$ & $\begin{array}{l}42^{\circ} 60^{\prime} 84^{\prime \prime} \mathrm{N} \\
24^{\circ} 88^{\prime} 83^{\prime \prime} \mathrm{E}\end{array}$ & 444 & 10.10 .2018 & 71 \\
\hline
\end{tabular}

\subsection{RESULTS ON ANALYS OF REQUIREMENTS FOR DIFFERENT PLANTS ABOUT WETTING ABILITY OF SPRAYS IN PESTICIDES APPLYCATION}

Figures 2, 3 and 4 shows the wetting requirements of the leaves of different cultures in four different growing stages.

From presented results above can be clearly seen that wetting ability of the sprayed liquid does not depend on plant surface structures wax content, but on the roughness of plant surface texture. The cabbage leaves although have a high wax content but are relative smooth (Lee et al., 1988), require to be sprayed with solutions with surface tension $25 \mathrm{mN} \mathrm{m}^{-1}$ for full wetting (Figure 4). However, the bean leaves with less wax content but more rough textures for full wetting require solutions with surface tension $21 \mathrm{mN} \mathrm{m}^{-1}$ (Figure 4). The tomato leaves in earlier growing stages when are more rough require solutions with $22 \mathrm{mN} \mathrm{m}^{-1}$ surface tension, while in late growing stages when they develop more smooth texture need to be sprayed with solutions with $30 \mathrm{mN} \mathrm{m}^{-1}$ surface tension. The leaves of apples (Malus domestica Borkh.), potato (Solanum tuberosum L.), maize (Zea mays L.) and cauliflower (Brassica oleracea L. ssp. oleracea convar. Botrytis (L.) Alef) which are relativelly smooth in all phenophases require to be sprayed with solutions with surface tension close to $22 \mathrm{mN} \mathrm{m}^{-1}$ (Figure 3).
There is obvious difference between leaves of cabbage and cauliflower, leaves of the second have more rough texture and for full wetting required solutions with lower surface tension. The test with bark also reveals that for good wetting of more rough textured plant surfaces - liquids with lower surface tension is required.

\subsection{RESULTS OF ANALYSIS OF IMPACT OF WA- TER SURFACE TENSION ON NOZZLE FLOW RATE}

Figure 5 shows flow rates obtained with Matabi sprayer with solutions with different surface tensions.

The nozzle of the sprayer was adjusted to spray with droplets with the size of 25 and 300 microns (diameter). The size of the droplets was visually determined by water sensitive papers and digital micrometer. The difference is obvious - the same sprayer with the same nozzle adjusted to a constant position and sprayed droplets with the same size but with solutions with different surface tension (wetting ability) achieved different flow rates. When sprayer works with distilled water $\left(66 \mathrm{mN} \mathrm{m}^{-1}\right.$ surface tension) $500 \mathrm{ml} \mathrm{m}^{-1}$ flow rate was achieved. The same sprayer, with the same nozzle adjustment working with the same water but with addition of organosilicone surfactant Silwet L 77 at 0.01 $\%$ concentration $\left(22 \mathrm{mN} \mathrm{m}^{-1}\right.$ surface tension), achieved 
$900 \mathrm{ml} \mathrm{m}^{-1}$ flow rate $-80 \%$ more solution pass through nozzle. With increase of the droplet size from 25 to 300 microns, and addition of organosilicone surfactant Silwet L 77 to the distilled water again at $0.01 \%(22$ $\mathrm{mN} \mathrm{m}^{-1}$ surface tension ), only $37 \%$ more solution pass from the nozzle.
In both cases (25 and 300 microns size of droplets) the addition of the same amount of organosilicone surfactant $(0.01 \%$ concentration $)$ to the distilled water decreased the surface tension to the same level (22 $\mathrm{mN} \mathrm{m^{-1 }}$ ) and dramatically increased the flow rate of the sprayer nozzle.

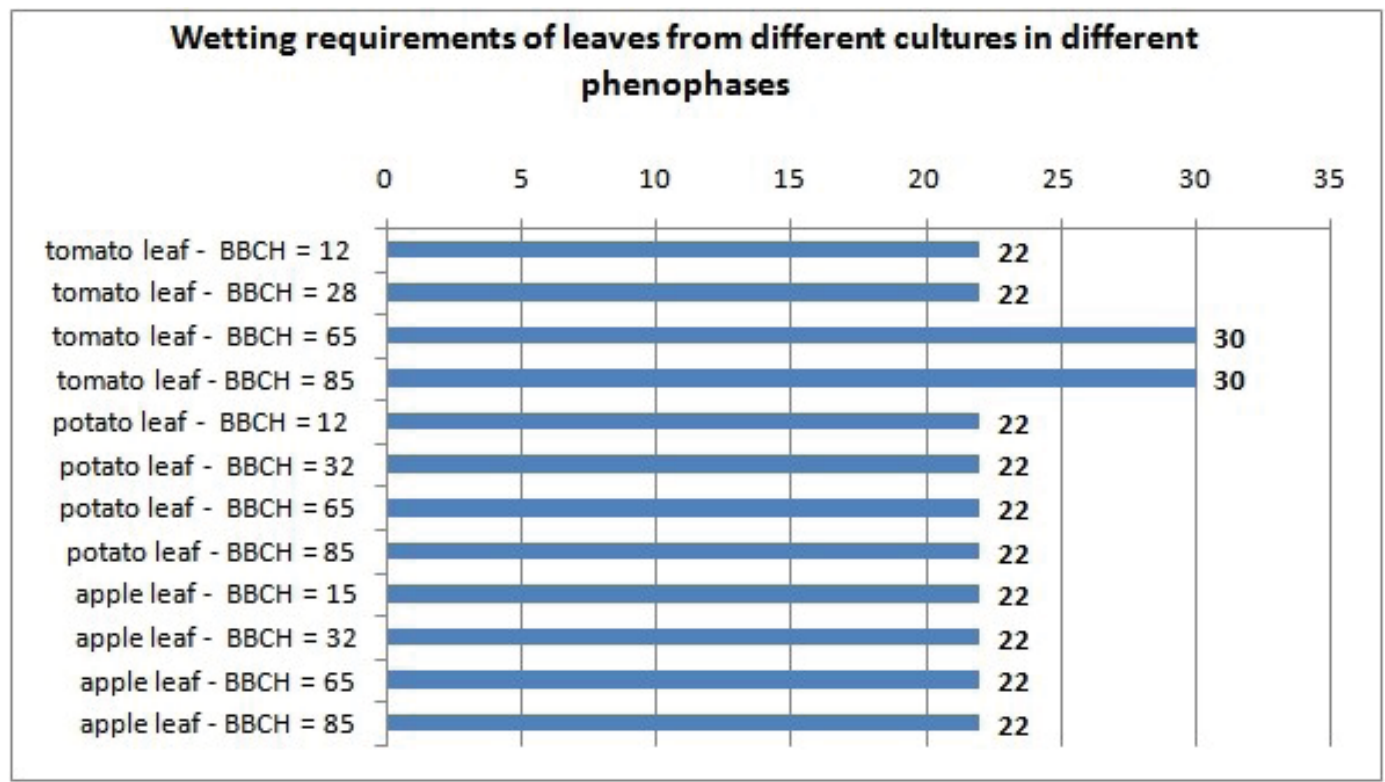

Figure 2: Wetting ability requirements of the crop leaves - on y axis are presented different plant leaves taken in different growth stages $\left(\mathrm{BBCH}\right.$ stages); on $\mathrm{x}$ axis is measured surface tension $\left(\mathrm{mN} \mathrm{m}^{-1}\right)$ of pesticides spray needed for full coverage and penetration in the texture of the leaves.

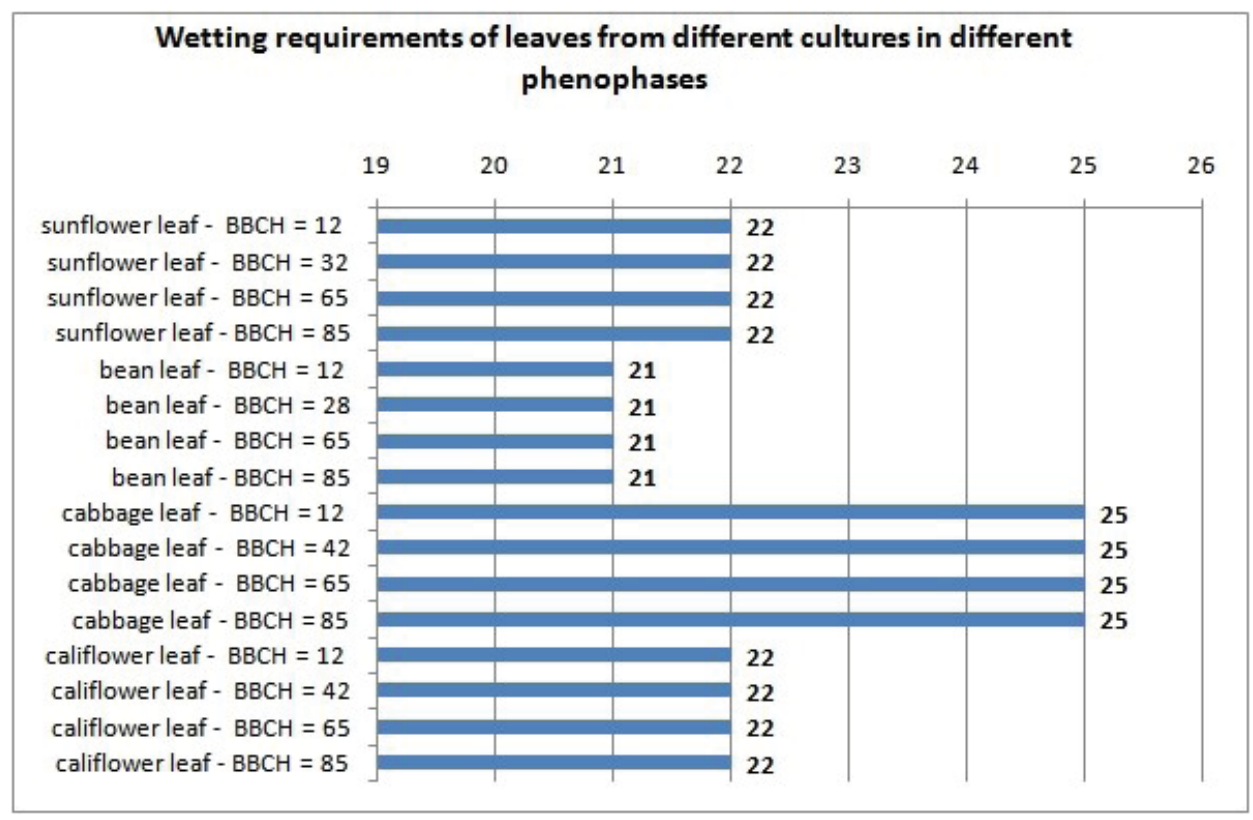

Figure 3: Wetting ability requirements of the crop leaves - on y axis are presented different plant leaves taken in different growth stages (BBCH stages); on $\mathrm{x}$ axis is measured surface tension $\left(\mathrm{mN} \mathrm{m}^{-1}\right)$ of pesticides spray needed for full coverage and penetration in the texture of the leaves 


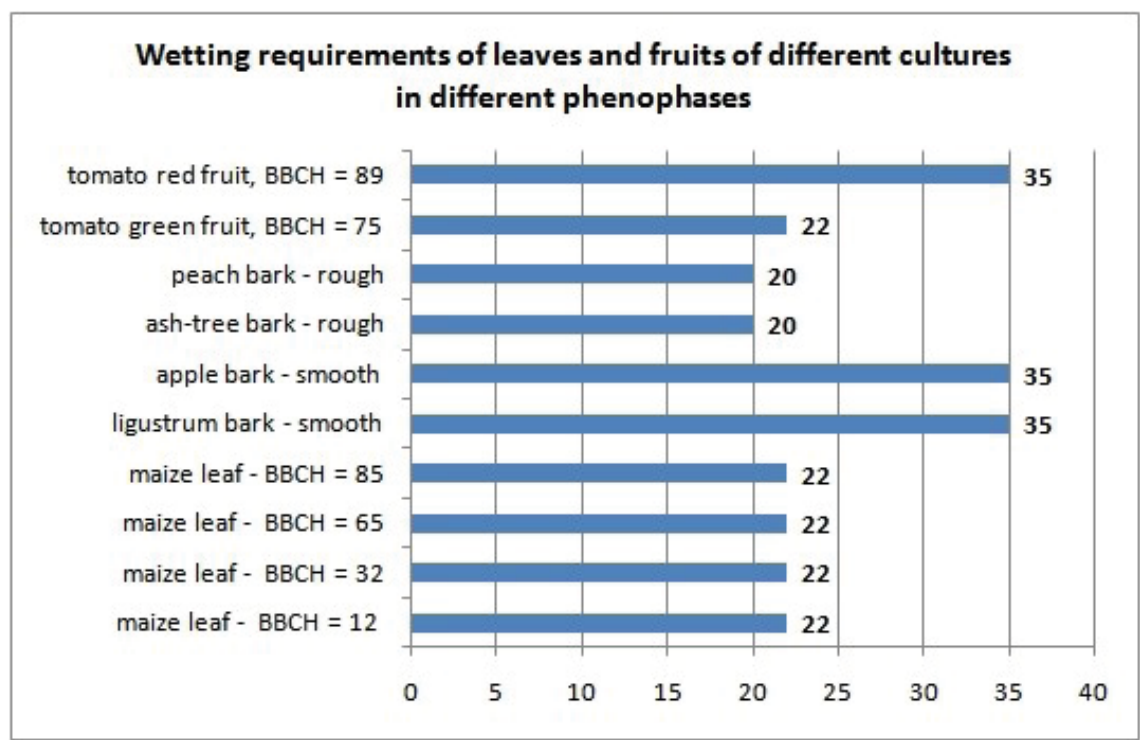

Figure 4: Wetting ability requirements of the crop leaves - on y axis are presented different plant leaves taken in different growth stages (BBCH stages); on $\mathrm{x}$ axis is measured surface tension $\left(\mathrm{mN} \mathrm{m}^{-1}\right)$ of pesticides spray needed for full coverage and penetration in the texture of the leaves

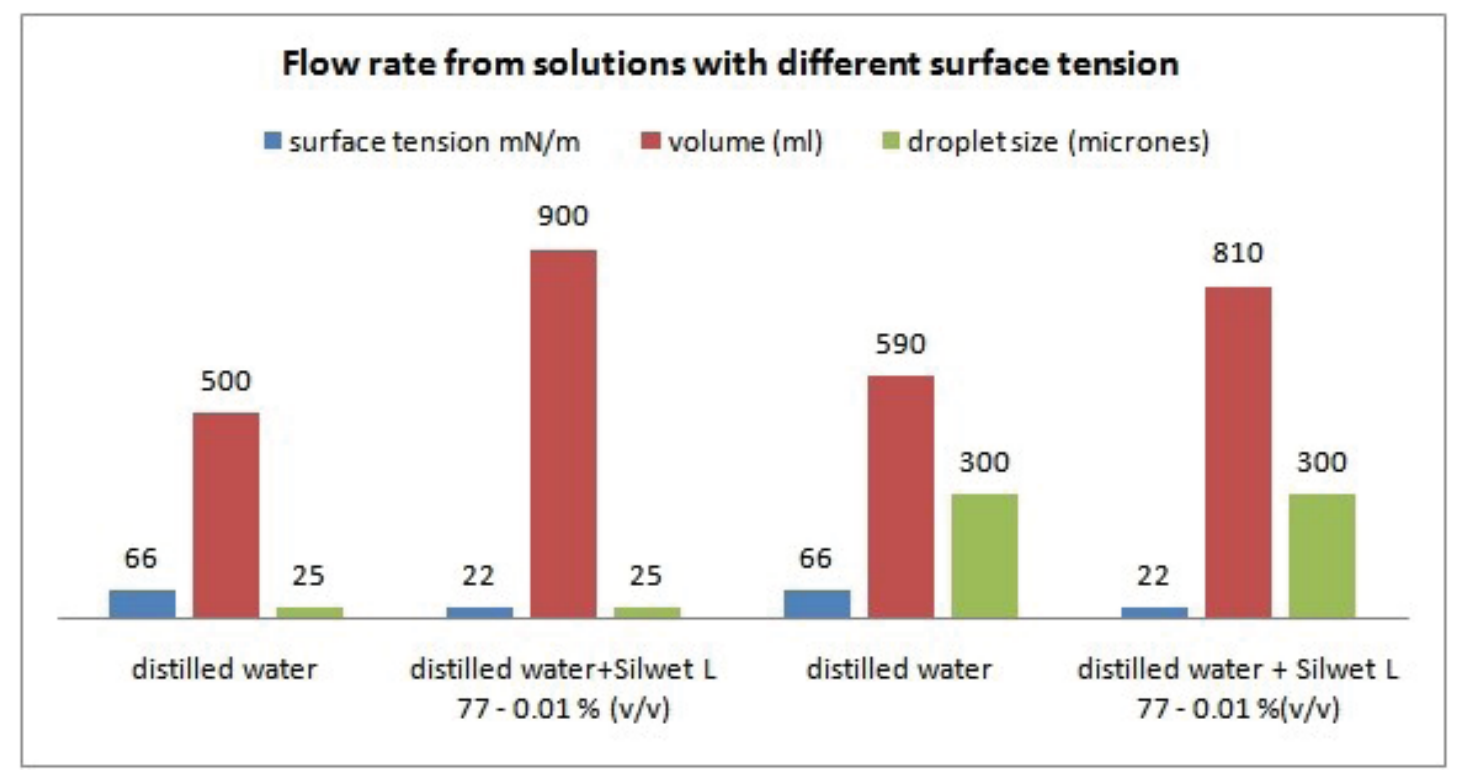

Figure 5: Flow rates $\left(\mathrm{ml} \mathrm{min}^{-1}\right)$ of solutions with different surface tensions - left two columns small droplets $(25 \mu \mathrm{m})$ and right two big droplets $(300 \mu \mathrm{m})$

\section{CONCLUSION}

The conducted tests proved that surface tension of the water used for preparation of the pesticide spray was not of constant value and depended not only from temperature, but also on many other factors and could be completely different from different sources and time periods (season, weather) without any logical correlation with location, time and type of water source.
Sprayed surfaces with more rough texture require to be treated with pesticide solutions with lower surface tension. The solutions with lower surface tension give a bigger nozzle flow rates than solutions with higher surface tension, especially when spray consists of smaller size droplets. The addition of wetting agent to the pesticide solutions can dramatically increase the flow rate from the sprayer. 


\section{REFERENCES}

Bartell, F. E., \& Zuidema, H. H. (1936). Wetting characteristics of solids of low surface tension such as talc, waxes and resins. Journal of the American Chemical Society, 58(8), 1449-1454. https://doi.org/10.1021/ja01299a041

Beattie, J. K., Djerdjev, A. M., Gray-Weale, A., Kallay, N., Lützenkirchen, J., Preočanin, T., \& Selmani, A. (2014). pH and the surface tension of water. Journal of Colloid and Interface Science, 422, 54-57. https://doi.org/10.1016/j. jcis.2014.02.003

Bergström, L. (1990). Use of lysimeters to estimate leaching of pesticides in agricultural soils. Environmental Pollution, 67(4), 325-347. https://doi.org/10.1016/02697491(90)90070-S

Grisso, R. D., Hewett, E. J., Dickey, E. C., Schnieder, R. D., \& Nelson, E. W. (1988). Calibration accuracy of pesticide application equipment. Applied Engineering in Agriculture, 4(4), 310-315. https://doi.org/10.13031/2013.26624

Bonn, D., Eggers, J., Indekeu, J., Meunier, J., \& Rolley, E. (2009). Wetting and Spreading. Reviews of Modern Physics, 81(2), 739. https://doi.org/10.1103/RevModPhys.81.739

Cerruto, E., Failla, S., Longo, D., \& Manetto, G. (2016). Simulation of water sensitive papers for spray analysis. Agricultural Engineering International: CIGR Journal, 18(4), 22-29.

Claussen, W. F. (1967). Surface tension and surface structure of water. Science, 156(3779), 1226-1227. https://doi. org/10.1126/science.156.3779.1226

Crease, G. J., Hall, F. R., \& Thacker, J. R. M. (1991). Reflection of agricultural sprays from leaf surfaces. Journal of Environmental Science \& Health Part B, 26(4), 383-407. https://doi.org/10.1080/03601239109372744

De Schampheleire, M., Nuyttens, D., Baetens, K., Cornelis, W., Gabriels, D., \& Spanoghe, P. (2009). Effects on pesticide spray drift of the physicochemical properties of the spray liquid. Precision Agriculture, 10(5), 409-420. https://doi. org/10.1007/s11119-008-9089-6

Ellis, M. B., Tuck, C. R., \& Miller, P. C. H. (2001). How surface tension of surfactant solutions influences the characteristics of sprays produced by hydraulic nozzles used for pesticide application. Colloids and Surfaces A: Physicochemical and Engineering Aspects, 180(3), 267-276. https://doi.org/10.1016/S0927-7757(00)00776-7

Gittens, G. J. (1969). Variation of surface tension of water with temperature. Journal of Colloid and Interface Science, 30(3), 406-412. https://doi.org/10.1016/0021-9797(69)90409-3

Hess, F. D., \& Foy, C. L. (2000). Interaction of Surfactants with Plant Cuticles1. Weed Technology, 14(4), 807-813. https:// doi.org/10.1614/0890-037X(2000)014[0807:IOSWPC]2.0 .CO;2

Kalová, J., \& Mareš, R. (2015). Reference values of surface tension of water. International Journal of Thermophysics, 36(7), 1396-1404. https://doi.org/10.1007/s10765-015-1907-2

Lee, C. H., Hwang, I. J., \& Kim, J. K. (1988). Macro-and microstructure of Chinese cabbage leaves and their texture measurements. Korean Journal of Food Science and Technology, 20(6), 742-748. https://doi.org/10.9721/ KJFST.2011.43.6.742

Macy, R. (1935). Surface tension by the ring method. Appli- cability of the du Nouy apparatus. Journal of Chemical Education, 12(12), 573 https://doi.org/10.1021/ed012p573.

Matthews, G. (2008). Pesticide application methods. John Wiley \& Sons.

Miller, P. C. H., \& Ellis, M. B. (2000). Effects of formulation on spray nozzle performance for applications from groundbased boom sprayers. Crop Protection, 19(8-10), 609-615. https://doi.org/10.1016/S0261-2194(00)00080-6

Nayar, K. G., Panchanathan, D., McKinley, G. H., \& Lienhard, J. H. (2014). Surface tension of seawater. Journal of Physical and Chemical Reference Data, 43(4), 043103. https://doi. org/10.1063/1.4899037

Pallas, N. R., \& Pethica, B. A. (1983). The surface tension of water. Colloids and Surfaces, 6(3), 221-227. https://doi. org/10.1016/0166-6622(83)80014-6

Pockels, A. (1893). Relations between the surface-tension and relative contamination of water surfaces. Nature, 48, 152 154. https://doi.org/10.1038/048152a0

Prado, E. P., Raetano, C. G., do Amaral Dal, M. H. F., Chechetto, R. G., Ferreira Filho, P. J., Magalhaes, A. C., \& Miasaki, C. T. (2016). Effects of agricultural spray adjuvants in surface tension reduction and spray retention on Eucalyptus leaves. African Journal of Agricultural Research, 11(40), 3959-3965. https://doi.org/10.5897/AJAR2016.11349

Salyani, M., \& Fox, R. D. (1999). Evaluation of spray quality by oiland water-sensitive papers. Transactions of the ASAE, 42(1), 37. https://doi.org/10.13031/2013.13206

Semiao, V., Andrade, P., \& da GraCa Carvalho, M. (1996). Spray characterization: numerical prediction of Sauter mean diameter and droplet size distribution. Fuel, 75(15), 17071714. https://doi.org/10.1016/S0016-2361(96)00163-9

Sridhar, M. K. C., \& Reddy, C. R. (1984). Surface tension of polluted waters and treated wastewater. Environmental Pollution Series B, Chemical and Physical, 7(1), 49-69. https://doi.org/10.1016/0143-148X(84)90037-5

Stevens, P. J. G., Gaskin, R. E., \& Zabkiewicz, J. A. (1988). Silwet L-77: a new development in spray adjuvants. In Proceedings of the New Zealand Weed and Pest Control Conference (Vol. 41, pp. 141-145). https://doi.org/10.30843/ nzpp.1988.41.9880

Tschapek, M., Scoppa, C. O., \& Wasowski, C. (1978). The surface tension of soil water. Journal of Soil Science, 29(1), 1721. https://doi.org/10.1111/j.1365-2389.1978.tb02026.x

Turner, C. R., \& Huntington, K. A. (1970). The use of a water sensitive dye for the detection and assessment of small spray droplets. Journal of Agricultural Engineering Research, 15(4), 385-387. https://doi.org/10.1016/00218634(70)90099-5

Wagner, P., Fürstner, R., Barthlott, W., \& Neinhuis, C. (2003). Quantitative assessment to the structural basis of water repellency in natural and technical surfaces. Journal of Experimental Botany, 54(385), 1295-1303. https://doi. org/10.1093/jxb/erg127

Yuan, Y., \& Lee, T. R. (2013). Contact angle and wetting properties. In Surface science techniques (pp. 3-34). Springer, Berlin, Heidelberg. https://doi.org/10.1007/9783-642-34243-1_1

Zhu, F., Cao, C., Cao, L., Li, F., Du, F., \& Huang, Q. (2019).

Wetting behavior and maximum retention of aqueous 
